\title{
FREQUENCIES OF APOLIPOPROTEINS E5 AND E7 IN APPARENTLY HEALTHY JAPANESE
}

\author{
Shigeru Tsuchiya, ${ }^{1}$ Yasuko Yamanouchi, ${ }^{2}$ Mieko Onuki, ${ }^{2}$ Kimiko Yamakawa, ${ }^{2}$ \\ Ryunosuke MiYaZaKi, ${ }^{3}$ Toshimitsu TAYA, ${ }^{4}$ Ikuko Kondo, ${ }^{2}$ \\ Minoru OHNuki, ${ }^{1}$ and Hideo HamaguchI ${ }^{2}$ \\ ${ }^{1}$ Department of Nursing and Rehabilitation Medicine, Institute of \\ Community Medicine, and \\ ${ }^{2}$ Department of Human Genetics, Institute of Basic Medical Sciences, \\ University of Tsukuba, Sakura-mura, Ibaraki 305, Japan \\ ${ }^{3}$ Kudanzaka-Hospital, Kudan-minami, Chiyoda-ku, Tokyo 102, Japan \\ ${ }^{4}$ Health Care Center, Tsuchiura-Kyodo Hospital, \\ Tsuchiura, Ibaraki 300, Japan
}

Summary Human apolipoprotein (apo) E plays an important role in the metabolism of cholesterol and other lipids. Apo E5 and apo E7 are genetic variants of apo $\mathrm{E}$ and have been detected in about $5 \%$ of Japanese patients with hyperlipidemia and ischemic heart disease. The existence of apo E5 and apo E7, however, had not been reported in apparently healthy individuals except for a few family members of the patients with apo E5 or apo E7. It has been suggested that apo E5 and apo E7 are closely related to the development of atherosclerosis. The purpose of this study is to investigate the frequency of apo E5 and apo E7 in apparently healthy Japanese and to analyze serum lipid levels of the individuals with apo E5 or apo E7.

The apo E phenotypes of 197 apparently healthy Japanese adults were determined by two-dimensional gel electrophoresis. The gene frequencies of apo E were: $\varepsilon 3,0.843 ; \varepsilon 4,0.112 ; \varepsilon 2,0.038 ; \varepsilon 5,0.006 ; \varepsilon 7,0.0035$. Three out of 187 subjects $(1.5 \%)$ were found to have apo E5 or apo E7 in heterozygous state. Two of them were heterozygous with apo E3 (apo E3/5 and apo E3/7) and the both had normal serum lipid levels, though they were more than 50 years old. The other individual was heterozygous with apo E2 (apo E2/5) and had mild hypertriglyceridemia. As to myocardial infarction, angina pectoris and cerebral infarction, no clinically abnormal findings were detected in all the three individuals with apo E5 or apo E7.

The data suggest that the frequency of the individuals with apo E5 or apo E7 is of the order of $1 \%$ and much higher than that of the homozygotes with apo E2/2 in Japanese. The data also indicate that further genetic, epidemiologic and clinical studies are required to determine whether $=5$ and $\varepsilon 7$ act as a dominant major gene, as a recessive major gene, or as 
one of polymeric genes, in predisposing one to hyperlipidemia and atherosclerosis.

\section{INTRODUCTION}

Apolipoprotein (Apo) E is a major protein constituent of human plasma very low density lipoproteins (VLDL) (Shelburne and Quarfordt, 1974). It is also a normal constituent of chylomicrons and a subfraction of the high density lipoproteins (HDL) (Weisgraber and Mahley, 1978; Mahley and Innerarity, 1983). Human apo $\mathrm{E}$ is a single chain polypeptide (Mr 34,000) of 299 amino acids and its complete amino acid sequences are known (Rall et al., 1982b). The locus for human apo E is closely linked to the loci for apo CII and the Lutheran blood group on chromosome 19 (Myklebost et al., 1984; Gedde-Dahl et al., 1984). Recently, the complete nucleotide sequence of the human apo E gene has been determined (Paik et al., 1985). The major function of apo $E$ is its mediation of the cellular uptake of specific plasma lipoproteins through an interaction with apo B,E (LDL) receptor on extrahepatic and hepatic cell surface and with distinct hepatic apo E receptor (Mahley and Innerarity, 1983). In addition, apo $E$ is involved in the normal conversion of VLDL to the low density lipoproteins (LDL) (Ehnholm et al., 1984). Thus, apo E plays an important role in the metabolism of cholesterol and other lipids.

In man, the locus for apo $\mathrm{E}$ is polymorphic (Utermann et al., 1977; Zannis et al., 1981; Zannis et al., 1982). Three common alleles, designated $\varepsilon 2, \varepsilon 3$ and $\varepsilon 4$, have been demonstrated to exist in various populations (Utermann et al., 1982; Wardell et al., 1982; Ghiselli et al., 1982; Cumming and Robertson, 1984; Asakawa et al., 1985; Sing and Davignon, 1985). The three common alleles, $\varepsilon 2$, $\varepsilon 3$ and $\varepsilon 4$, code for three common apo $\mathrm{E}$ isoforms, apo E2, apo E3 and apo E4, respectively and determine six apo E phenotypes (Zannis et al., 1981; Zannis et al., 1982; Utermann et al., 1982). Futhermore, rare mutant genes have been described (Chiselli et al., 1982; Weisgraber et al., 1984; Gregg et al., 1983; Yamamura et al., 1984a; Yamamura et al., 1984b). Besides an association of apo E2 with type III hyperlipidemia (Havel, 1982), most of apo $\mathrm{E}$ isoforms encoded by these rare mutant genes have been reported to be associated with hyperlipidemia or atherosclerotic diseases or both. They include apo E Bethesda (apo E1) detected in a Caucasian family and apo E5 and apo E suita (apo E7) found in Japanese families (Gregg et al., 1983; Yamamura et al., 1984a; Yamamura et al., 1984b). Apo E5 and apo E7 have been thus far detected only in Japanese patients with myocardial infarction and hyperlipidemia and in their family members (Yamamura et al., 1984b).

The main purpose of this study is to investigate the frequency of apo E5 and apo E7 in apparently healthy Japanese. Our data suggest that the frequency of individuals with apo E5 or apo E7 is of the order of $1 \%$ and much higher than that of homozygotes with apo E2/2 in Japanese. 


\section{MATERIALS AND METHODS}

Subjects and samples. We studied 197 apparently healthy Japanese: 85 males and 8 females aged 35-64 years who visited the health care center of Kudanzaka hospital in Tokyo; 53 males aged 40-57 years who visited the health care center of Tsuchiura-kyodo hospital in Tsuchiura city; 51 staffs (30 males and 21 females), aged 22-43 years, of University of Tsukuba. Blood samples were taken after an overnight fast.

Laboratory procedures. Ten $\mu$ l of VLDL-rich fraction was separated from 175 $\mu l$ of serum by ultracentrifugation for $3 \mathrm{hr}$ at $160,000 \times g$ using Beckmann Airfuge Ultracentrifuge (Beckman Instruments, Inc., Irvine, California). To determine the phenotype of apo E, $10 \mu \mathrm{l}$ of VLDL-rich fraction was subjected to two-dimentional (two-D) gel electrophoresis. Two-D gel electrophoresis was performed essentially according to the method of O'Farrell (1977) as previously described (Hamaguchi et al., 1982) with minor modifications: Ampholine mixtures of $1.4 \% \mathrm{pH}$ range 5-8 and $0.6 \% \mathrm{pH}$ range 3-10 were used in the first dimentional disc gel. After two-D gel electrophoresis, polypeptides were visualized on slab gels by the silver stain technique of Merril et al. (1981). The apo E spots on slab gels were identified by the method of immuno-blotting (Towbin et al., 1979), using goat anti-human apo E (Daiichi Pure Chemicals Ltd., Tokyo) and fluorescein-conjugated rabbit anti-goat IgG (Cappel Laboratories Inc., Malvern, Pennsylvania). Serum total cholesterol and triglyceride were measured enzymatically (Allain et al., 1974; Sampson et al, 1975): The samples from Kudanzaka hospital were analyzed on an automated analyzer (Hitachi Model 712, Hitachi Ltd., Japan) and the other samples manually Chemicals using commercial kits (Cholesterol E-Test and Triglyceride CII-Test, Wako Pure Ltd., Tokyo).

\section{RESULTS}

Figure I shows the two-D gel electrophoresis pattern of the three most common apo E phenotypes, apo E3/3, apo E3/4 and apo E2/3. In this survey, apo E5 was found in heterozygous state with apo E2 or apo E3 in two males. Figure 2 shows the two-D gel electrophoresis pattern of apo E $2 / 5$ phenotype, together with the data on the identification of the apo E spot on the gel by immuno-blotting. In addition to apo E5, apo E7 was also found in heterozygous state with apo E3 in one male. The two-D gel electrophoresis pattern of apo E3/7 phenotype is shown in Fig. 3. The apo E7 spot on the slab gel was also indentified as apo E by immunoblotting (data not shown). No apo El was found.

Table 1 presents the distribution of apo E phenotypes in 197 subjects. The gene frequencies of apo $\mathrm{E}$ are: $\varepsilon 3,0.843 ; \varepsilon 4,0.112 ; \varepsilon 2,0.038 ; \varepsilon 5,0.005 ; \varepsilon 7,0.0035$. It can be seen that the observed numbers are close to those calculated on the assump- 

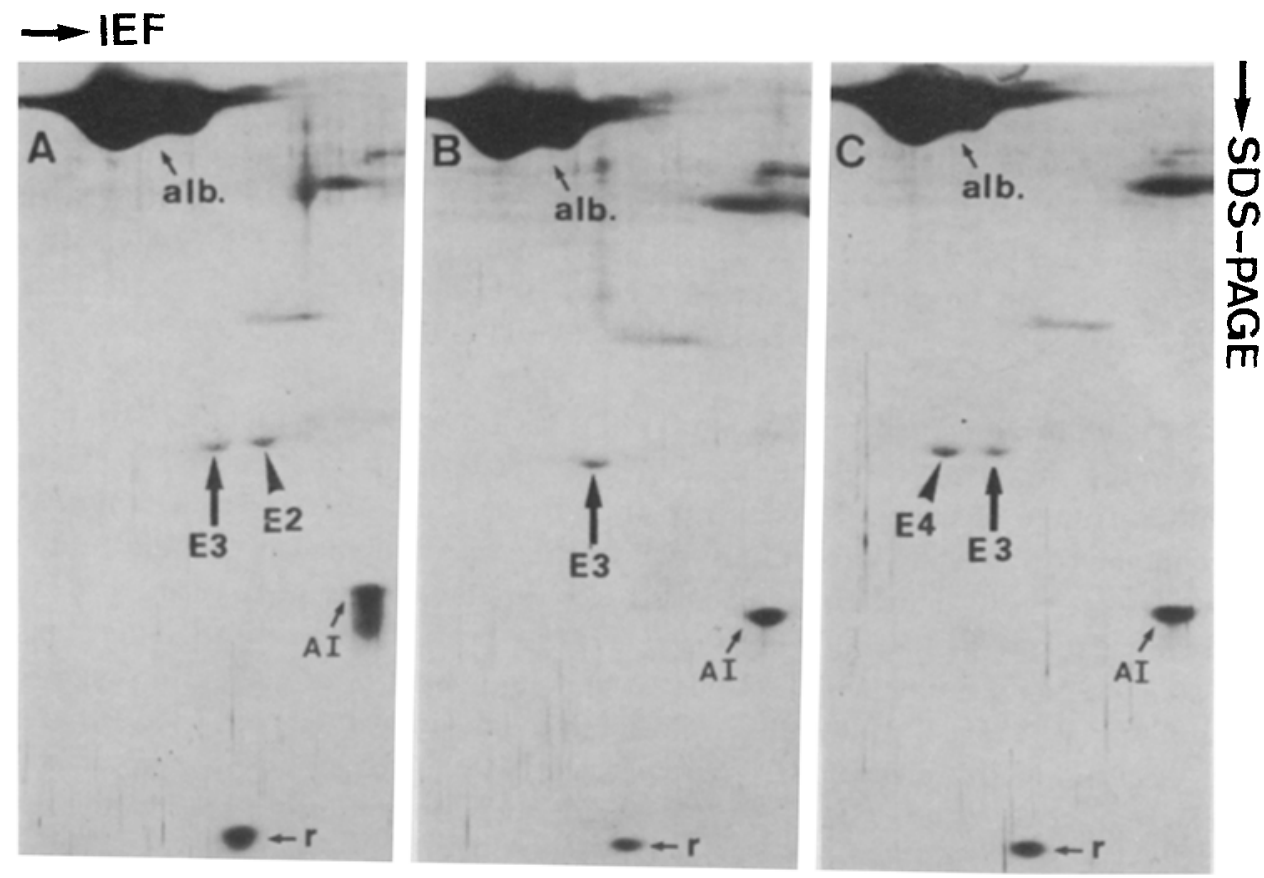

Fig. 1. Two-D gel electrophoresis patterns of the three most common apo $E$ phenotypes. $\mathrm{A}$, apo E2/3; B, apo E3/3; C, apo E3/4. Apo E2, apo E3, and apo E4 are indicated by E2, E3, and E4, respectively. Albumin and apolipoprotein A-I are shown by alb and AI, respectively. The polypeptide indicated by $\mathrm{m}$ is a usefu! marker for the determination of apo $\mathrm{E}$ phenotypes. Isoelectric focusing was from left to right and molecular weight separation from top to bottom.

tion that Hardy-Weinberg equilibrium is observed. The frequencies of the three common alleles, $\varepsilon 2, \varepsilon 3$ and $\varepsilon 4$, are similar to those observed in Hiroshima (Asakawa et al., 1985). However, it is noteworthy that three out of 187 subjects $(1.5 \%)$ were found to have apo E5 or apo E7 in heterozygous state.

Table 2 shows the serum total cholesterol and triglyceride levels of the three subjects with apo E5 or apo E7. In two individuals with apo E3/5 and apo E3/7, the serum lipid levels were normal. On the other hand, one individual with apo E2/5 had mild hypertriglyceridemia.

\section{DISCUSSION}

Apo E5 and apo E7 have been detected in about $5 \%$ of Japanese patients with hyperlipidemia and ischemic heart disease, while they were not found among 100 healthy Japanese (Yamamura et al., 1984a; Yamamura et al., 1984b). We also found four cases with apo E5 or apo E7 $(4.3 \%)$ out of 92 patients with myocardial 

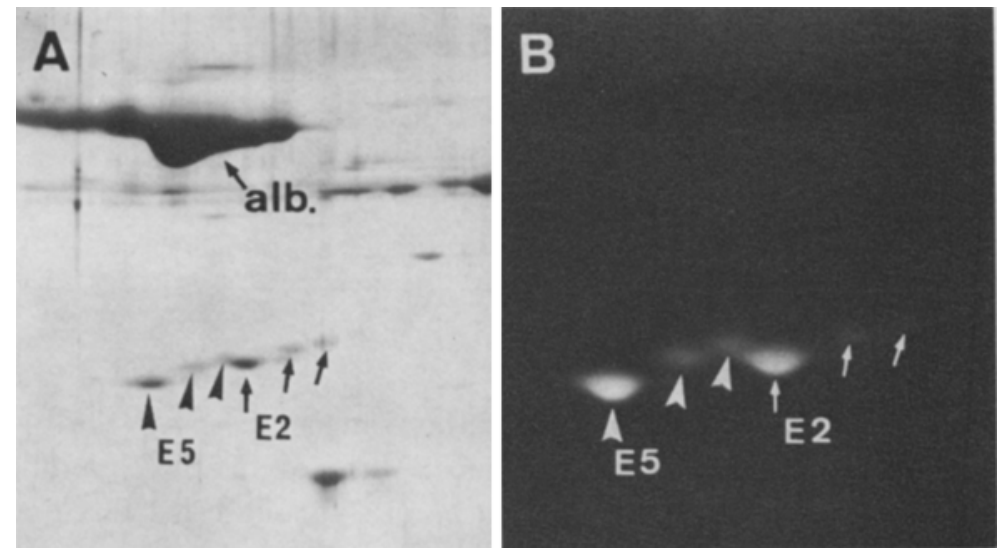

Fig. 2. Two-D gel electrophoresis pattern of apo E2/5. A, silver staining; B, immunoblotting. Apo E2 and apo E5 are indicated by E2 and E5, respectively. Albumin is shown by alb.
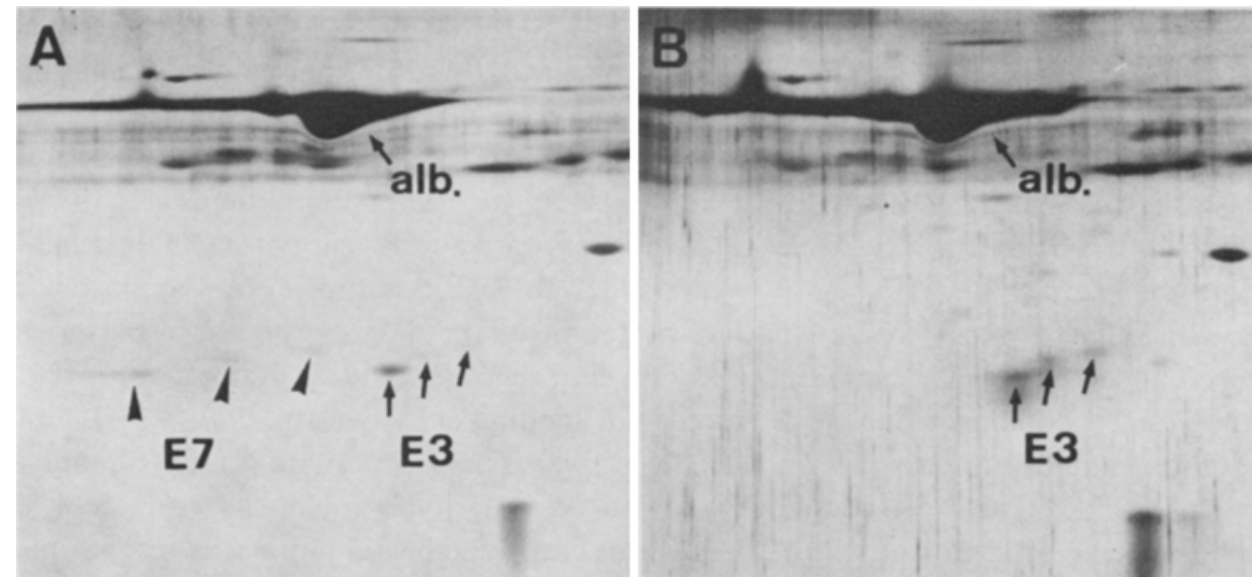

Fig. 3. Two-D gel electrophoresis pattern of apo E3/7 (A) and apo E3/3 (B). Apo E3 and apo E7 are indicated by E3 and E7, respectively. Albumin is shown by alb.

Table 1. Apo E phenotypes among 197 apparently healthy adults.

\begin{tabular}{lllllllll} 
& \multicolumn{8}{c}{ Phenotypes } \\
\cline { 2 - 8 } & $3 / 3$ & $3 / 4$ & $2 / 3$ & $4 / 4$ & $2 / 4$ & $3 / 5$ & $2 / 5$ & $3 / 7$ \\
\hline Observed & 141 & 37 & 11 & 2 & 3 & 1 & 1 & 1 \\
Expected & 139.9 & 37.1 & 12.6 & 2.4 & 1.6 & 1.6 & 0.07 & 0.9 \\
\hline
\end{tabular}

Expected values calculated from gene frequencies: $\varepsilon 3=0.843, \varepsilon 4=0.111, \varepsilon 2=0.038, \varepsilon 5=0.005$, and $\varepsilon 7=0.0025$. 
Table 2. Serum lipid values in subjects with apo E5 or apo E7.

\begin{tabular}{cccccc}
\hline Subject & $\begin{array}{c}\text { Age } \\
\text { (years) }\end{array}$ & Sex & $\begin{array}{c}\text { Apo E } \\
\text { phenotypes }\end{array}$ & $\begin{array}{c}\text { Cholesterol } \\
\text { (mg/dl) }\end{array}$ & $\begin{array}{c}\text { Triglyceride } \\
\text { (mg/dl) }\end{array}$ \\
\hline HS-T11 & 60 & M & $3 / 5$ & 174 & 116 \\
HS-T32 & 51 & M & $3 / 7$ & 231 & 154 \\
HS-K64 & 36 & M & $2 / 5$ & 178 & $188 \uparrow$ \\
\hline
\end{tabular}

Arrow indicates an increase in lipid value.

infarction (Hamaguchi et al., in preparation). Yamamura et al. (1984b) observed that five of seven patients with apo E5 or apo E7 had overt atherosclerotic disease. Based on their observations, Yamamura et al. (1984b) suggested that apo E5 and apo E7 are closely related to the development of atherosclerosis. In the present study, apo E5 and apo E7 were detected for the first time in apparently healthy subjects, with a frequency of $1.5 \%$. According to our data, the expected frequency of homozygotes with apo E2/2 is $0.14 \%$. Our data suggest that the frequency of individuals with apo E5 or apo E7 is of the order of $1 \%$ and much higher than that of homozygotes with apo E2/2 in Japanese.

Two of three individuals with apo E5 or apo E7 had normal values for plasma lipid levels, though they both were more than 50 years old. They are heterozygous with apo E3. Apo E3 has normal function in both receptor binding activities and the conversion of VLDL to LDL (Weisgraber et al., 1982; Ehnholm et al., 1984). On the other hand, the other individual with very rare apo E genotype, $\varepsilon 2 / 5$, had mild hypertriglyceridemia. He was 36 years old. In this case, however, the existence of $\varepsilon 2$ should be considered in the interpretation of the data. Apo E2 displays reduced apo B,E receptor binding activity (Weisgraber et al., 1982; Rall et al., 1982a; Rall et al., 1983) and reduced apo E receptor binding activity (Hui et al., 1984). In addition, apo E2 appears to impede the conversion of VLDL to LDL (Ehnholm et al., 1984). Usually $\varepsilon 2$ is associated with type III hyperlipidemia as a recessive gene. In the present case with $\varepsilon 2 / 5$, it is not clear whether $\varepsilon 5$ alone was responsible for hypertriglyceridemia or the combination of $\varepsilon 5$ with $\varepsilon 2$ was important as the etiology of hyperlipidemia. As to myocardial infarction, angina pectoris and cerebral infarction, no clinically abnormal findings were detected in all the three individuals with apo E5 or apo E7. Further genetic, epidemiologic and clinical studies as well as the analysis of functions are required to determine whether $\varepsilon 5$ and $\varepsilon 7$ act as a dominant major gene, as a recessive major gene, or as one of polymeric genes, in predisposing one to hyperlipidemia and atherosclerosis.

Acknowledgments This investigation was supported by a Scientific Research Grant from the Ministry of Education, Science and Culture of Japan, and a Research Grant for Intractable Diseases from the Ministry of Health and Welfare of Japan. We are grateful to Mr. Shiro Abe and Mr. Masaaki Teranishi for the preparation of photographs. 


\section{REFERENCES}

Allain, C.C., Poon, L.S., Chan, F.C.S., Richmond, W., and Fu, C.P. 1974. Enzymatic determination of total serum cholesterol. Clin. Chem. 20:470-475.

Asakawa, J., Takahashi, N., Rosenblum, B.B., and Neel, J.V. 1985. Two-dimensional gel studies of genetic variation in the plasma proteins of Amerindians and Japanese. Hum. Genet. 70: 222-230.

Chiselli, G., Schaefer, E.J., Gascon, P., and Brewer, H.B., Jr. 1981. Type III hyperlipidemia associated with apolipoprotein $\mathrm{E}$ deficiency. Science 214: 1239-1241.

Cumming, A.M. and Robertson, F.W. 1984. Polymorphism of the apoprotein-E locus in relation to risk of coronary disease. Clin. Genet. $25: 310-313$.

Ehnholm, C., Mahley, R.W., Chappell, D.A., Weisgraber, K.H., Ludwig, E., and Witzum, J.L. 1984. Role of apolipoprotein $\mathrm{E}$ in the lipolytic conversion of $\beta$-very low density lipoproteins to low density lipoproteins in type III hyperlipoproteinemia. Proc. Natl. Acad. Sci. U.S.A. 81: $5566 \rightarrow 5570$.

Gedde-Dahl, T., Jr., Olaisen, B., Teisberg, P., Wilhemly, M.C., Mevag, B., and Helland, R. 1984. The locus for apolipoprotein $\mathrm{E}$ is close to the Lutheran blood group locus on chromosome 19. Hum. Genet. 67: 178-182.

Ghiselli, G., Gregg, R.E., Zech, L.A., Schaefer, E.J., and Brewer, H,B., Jr. 1982. Phenotype study of apolipoprotein $\mathrm{E}$ isoforms in hyperlipoproteinemic patients. Lancet 2: 405-407.

Gregg, R.E., Chiselli, G., and Brewer, H.B., Jr. 1983. Apolipoprotein E Bethesda: A new variant of apolipoprotein E associated with type III hyperlipoproteinemia. J. Clin. Endocrinol. Metab. 57: 969-974.

Hamaguchi, H., Yamada, M., Shibasaki, M., Mukai, R., Yabe, T., and Kondo, I. 1982. Genetic analysis of human lymphocyte proteins by two-dimensional gel electrophoresis: 3 . Frequent occurrence of genetic variants in some abundant polypeptides of PHA-stimulated peripheral blood lymphocytes. Hum. Genet. 62: 142-147.

Havel, R.J. 1982. Familial dysbetalipoproteinemia. New aspects of pathogenesis and diagnosis. Med. Clin. North. Am. 66: 441-454.

Hui, D.Y., Innerarity, T.L., and Mahley, R.W. 1984. Defective hepatic lipoprotein receptor binding of $\beta$-very low density lipoproteins from type III hyperlipoproteinemic patients. Importance of apolipoprotein E. J. Biol. Chem. 259: 860-869.

Mahley, R.W. and Innerarity, T.L. 1983. Lipoprotein receptor and cholesterol homeostasis. Biochim. Biophys. Acta 737: 197-222.

Merril, C.R., Goldman, D., Sedman, S.A., and Ebert, M.H. 1981. Ultrasensitive stain for proteins in polyacrylamide gels show regional variation in cerebrospinal fluid proteins. Science 211: 1437-1438.

Myklebost, O., Ronge, S., Olaisen, B., Gedde-Dahl, T., Jr., and Prydz, H. 1984. The locus for apolipoprotein CII is closely linked to apolipoprotein $\mathrm{E}$ locus on chromosome 19 in man. Hum. Genet. 67: 309-312.

O'Farrell, P.H. 1975. High resolution two-dimensional electrophoresis of proteins. J. Biol. Chem. 250: $4007-4021$.

Paik, Y.-K., Chang, D.J., Reardon, C.A., Davies, G.E., Mahley, R.W., and Taylor, J.M. 1985. Nucleotide sequence and structure of the human apolipoprotein $\mathrm{E}$ genes. Proc. Natl. Acad. Sci. U.S.A. 82: 3445-3449.

Rall, S.C., Jr., Weisgraber, K.H., Innerarity, T.L., Bersot, T.P., Mahley, R.W., and Blum, C.B. 1983. Identification of a new structural variant of human apolipoprotein E, E2 (Lys146 $\rightarrow$ G1n), in a type III hyperlipoproteinemic subject with the E3/2 phenotype. J. Clin. Invest. 72: 1288-1297.

Rall, S.C., Jr., Weisgraber, K.H., Innerarity, T.L., and Mahley, R.W. 1982a. Structural basis for 
receptor binding heterogeneity of apolipoprotein E from type III hyperlipoproteinemic subjects. Proc. Natl. Acad. Sci. U.S.A. 79: 4696-4700.

Rall, S.C., Jr., Weisgraber, K.H., and Mahley, R.W. 1982b. Human apolipoprotein E. Complete amino acid sequence. J. Biol. Chem. 257: 4171-4175.

Sampson, E.J., Demers, L.M., and Krieg, A.F. 1975. Faster enzymatic procedure for serum triglycerides. Clin. Chem. 21: 1983-1985.

Shelburne, F.A. and Quarford, S.H. 1974. A new apolipoprotein of human plasma very low density lipoproteins. J. Biol. Chem. 249: 1428-1433.

Sing, C.E. and Davignon, J. 1985. Role of the apolipoprotein E polymorphism in determining normal plasma lipid and lipoprotein variation. Am. J. Hum. Genet. 37: 268-285.

Towbin, H., Staehelin, T., and Gordon, J. 1979. Electrophoretic transfer of proteins from polyacrylamide gels to nitrocellulose sheets: Procedure and some applications. Proc. Natl. Acad. Sci. U.S.A. 76: 4350-4354.

Utermann, G., Hees, M., Steinmets, A. 1977. Polymorphism of apolipoprotein E and occurrence of dysbetalipoproteinemia in man. Nature 269: 604-607.

Utermann, G., Steinmetz, A., and Weber, W. 1982. Genetic control of human apolipoprotein E polymorphism: Comparison of one- and two-dimensional techniques of isoprotein analysis. Hum. Genet. 60: 344-351.

Wardell, M.R., Suckling, P.A., and Janus, E.D, 1982. Genetic variation in human apolipoprotein E. J. Lipid. Res. 23: 1174-1182.

Weisgraber, K.H., Innerarity, T.L., and Mahley, R.W. 1982. Abnormal lipoprotein receptor-binding activity of the human $\mathrm{E}$ apoprotein due to cysteine-arginine interchange at a single site. J. Biol. Chem. 257: 2518-2521.

Weisgraber, K.H. and Mahley, R.W. 1978. Apoprotein (E-A-II) complex of human plasma lipoproteins. I. Characterization of the mixed disulfide and its identification in a high density lipoprotein subfraction. J. Biol. Chem. 253: 6281-6288.

Yamamura, T., Yamamoto, A., Hiramori, K., and Nambu, S. 1984a. A new isoform of apolipoprotein E. Apo ES associated with hyperlipidemia and atherosclerosis. Atherosclerosis 50: 159172.

Yamamura, T., Yamamoto, A., Sumiyoshi, T., Hiramori, K., Nishioeda, Y., and Nambu, S. 1984b. New mutants of apolipoprotein $\mathrm{E}$ associated with atherosclerotic diseases but not type III hyperlipidemia. J. Clin. Invest. 74: 1229-1237.

Zannis, V.I., Just, P.W., and Breslow, J.L. 1981. Human apolipoprotein E isoprotein subclasses are genetically determined. Am. J. Hum. Genet. 33, 11-24.

Zannis, V.I., Breslow, J.L., Utermann, G., Mahley, R.W., Weisgraber, K.H., Havel, R.J., Goldstein, J.L., Brown, M.S., Schonfeld, G., Hazzard, W.R., and Blum, C. 1982. Proposed nomenclature of apoE isoproteins, apoE genotypes, and phenotypes. J. Lipid. Res. 23: 911-914. 